\title{
Drought stress imposes reversible photosynthetic damage under fluctuating light conditions in crops
}

\author{
Kazuma Sakoda ${ }^{1}$, Kazuki Taniyoshi ${ }^{2}$, Wataru Yamori ${ }^{1}$, and Yu Tanaka ${ }^{2}$ \\ ${ }^{1}$ THE UNIVERSITY OF TOKYO \\ ${ }^{2}$ Kyoto University
}

February 8, 2021

\begin{abstract}
Drought stress is a major limiting factor for crop growth and yield. Water availability in the field can cyclically change between drought and rewatering conditions, depending on precipitation patterns. Concurrently, light intensity under field conditions can fluctuate, inducing dynamic photosynthesis and transpiration during crop growth period. The present study aimed to characterize carbon gain and water use in fluctuating light under drought and rewatering conditions by conducting gas exchange measurements in two major crops, namely rice and soybean. In both crops, drought stress reduced steady-state photosynthesis and/or photosynthetic capacity, and delayed photosynthetic induction even when it had relatively small impact on photosynthetic capacity, suggesting that the drought effects on photosynthesis should be evaluated based on induction, maximum, and steady states. This delayed photosynthetic induction resulted in a substantial loss of carbon gain under fluctuating light conditions, which can be a limiting factor for crop growth and yield in the field. Meanwhile, rewatering after drought conditions completely recovered photosynthetic capacity and induction in both crops, whereas drought experience would be memorized to slow down the stomatal opening. Therefore, the stability of photosynthetic induction can be a promising target to improve drought tolerance during crop breeding in the future.
\end{abstract}

\section{Hosted file}

Manuscript.pdf available at https://authorea.com/users/394569/articles/507919-drought-stressimposes-reversible-photosynthetic-damage-under-fluctuating-light-conditions-in-crops 


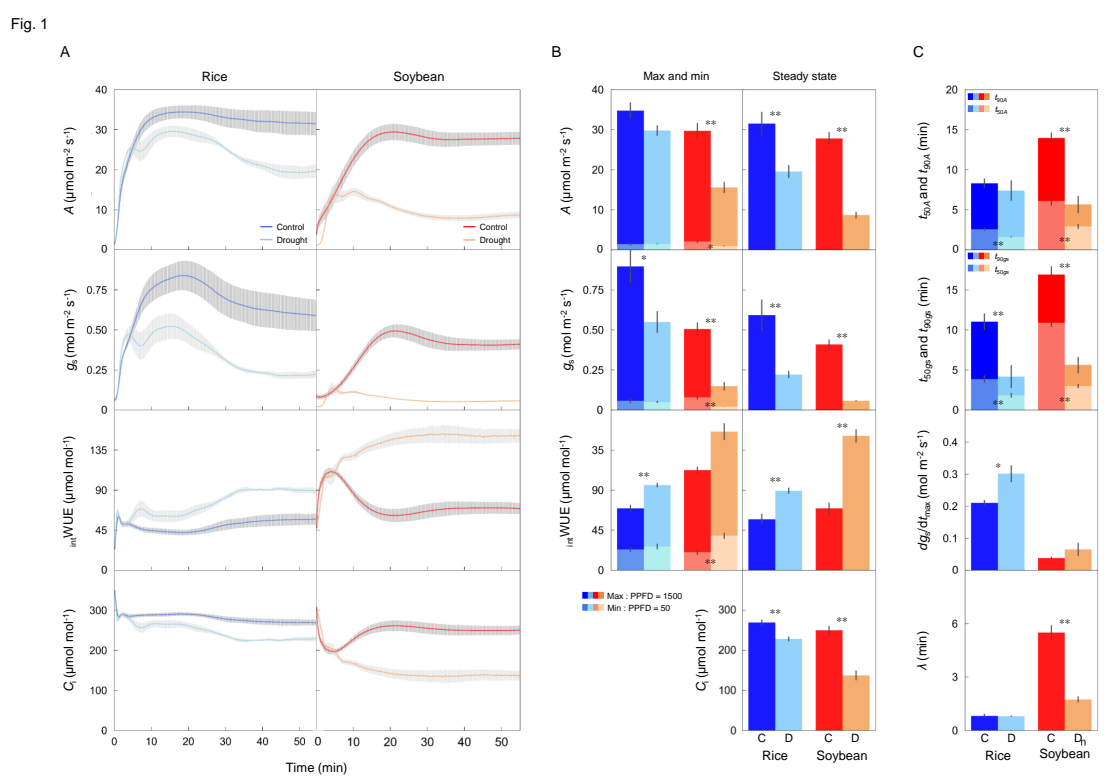

Fig. 2

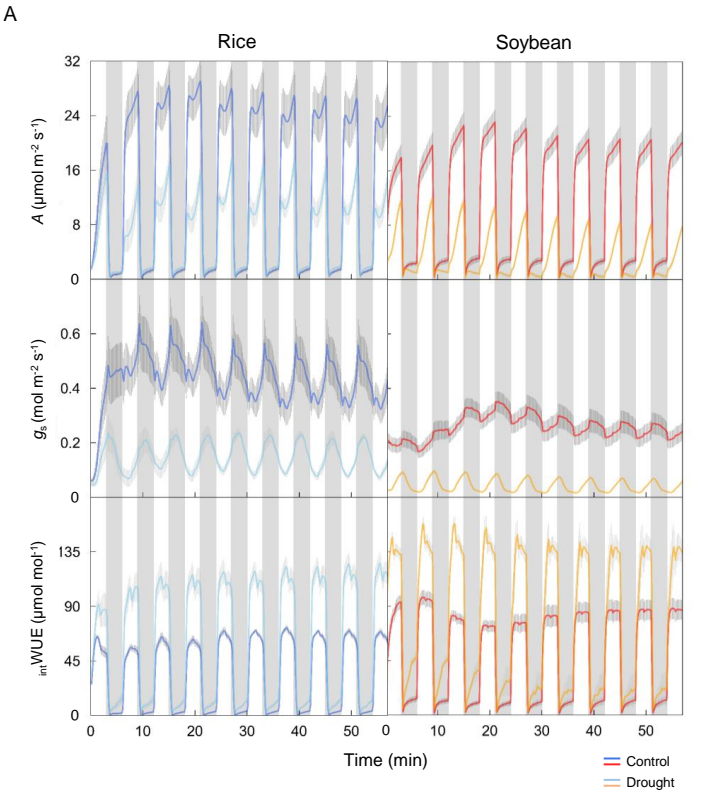

B
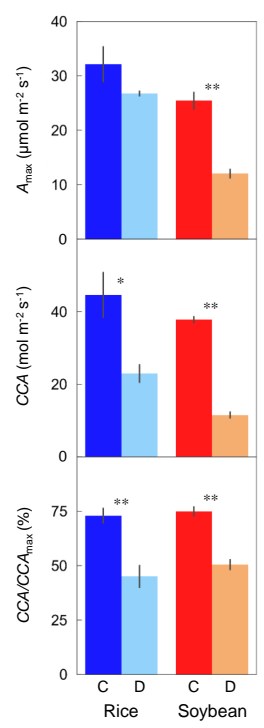

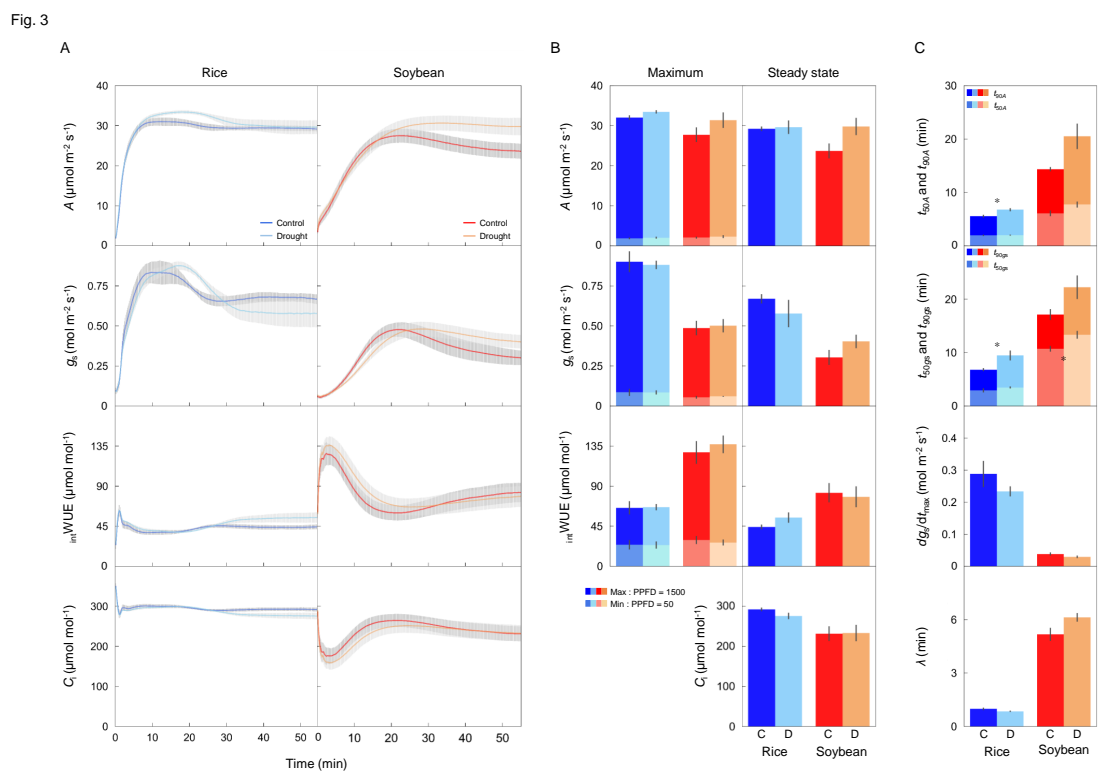

Fig. 4

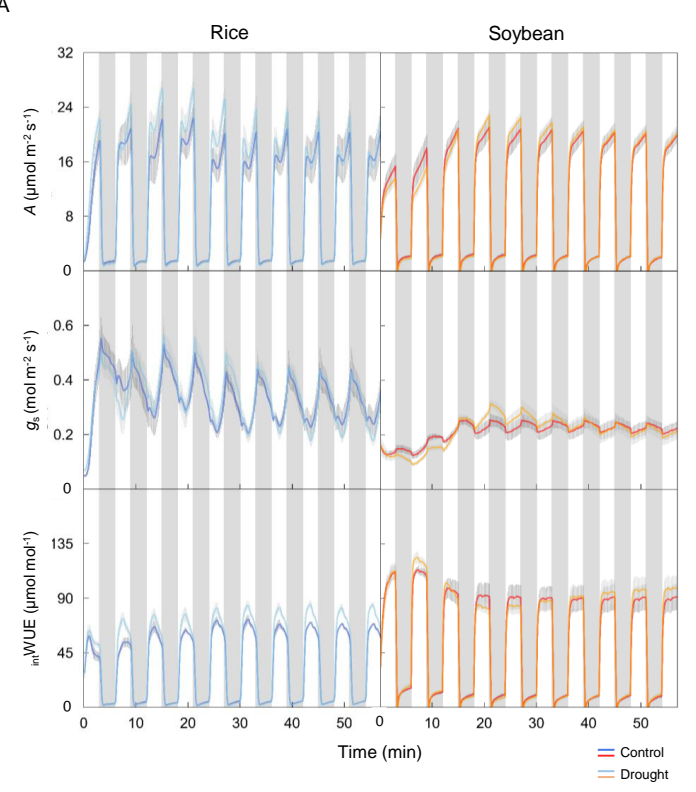

B
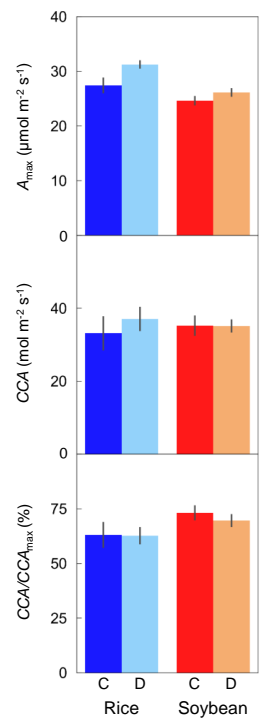
Fig. 5

A

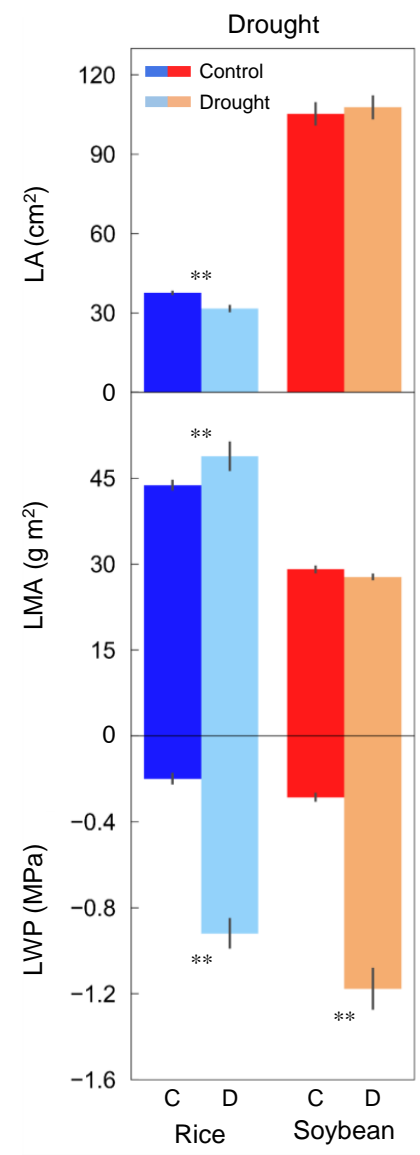

B

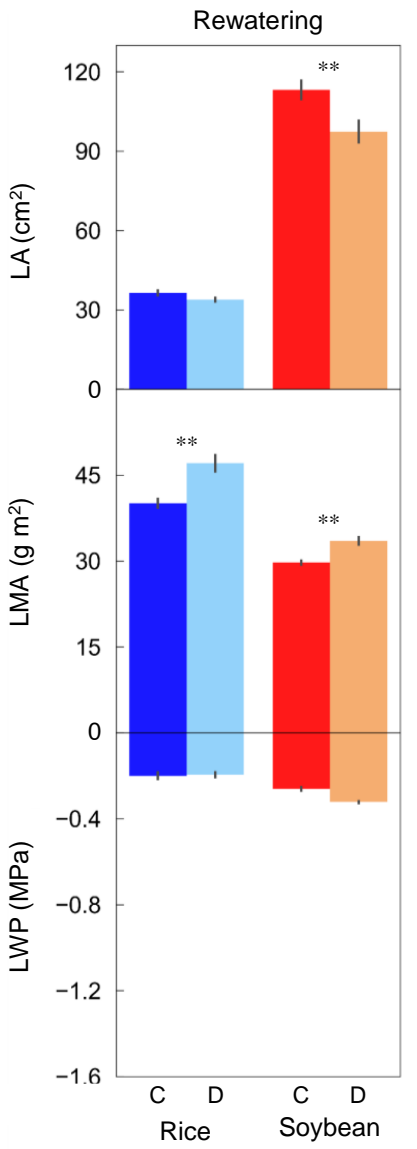


Fig. 6

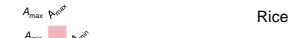

$A_{\text {matast }}=$

$g_{\max } * \sigma^{\prime}$

$g_{\text {minin }}+* * * *+\infty$

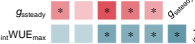

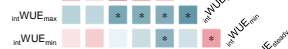

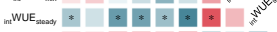

$c_{\text {atatar }}$

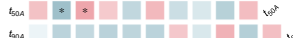

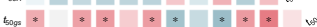

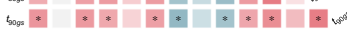

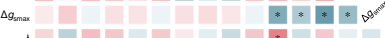

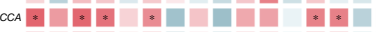

CCACCA $_{\operatorname{lax}} *$ * * * * * * *

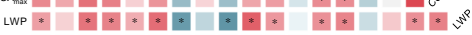

LMA * * * * * * *
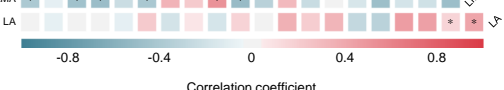

Correlation coefficient

C

12
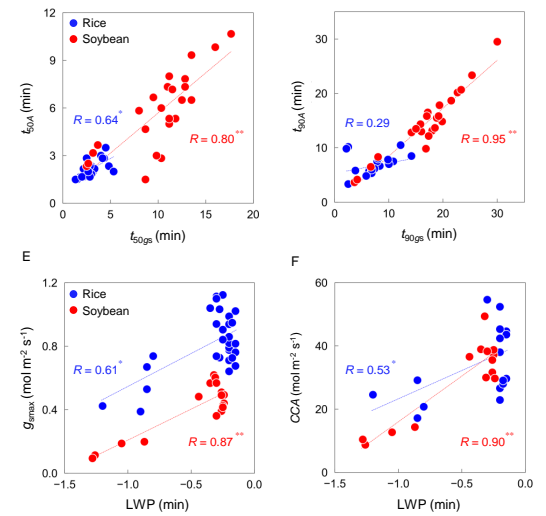

$A_{\max } \quad$ Soybean

$A_{\min }+\cdots$

$g_{\max } * * 0$

$g_{\min } * * * * *$

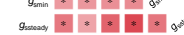

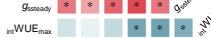

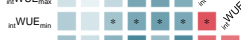

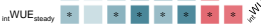

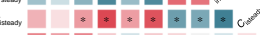

$\tan * * *+10$

bes ***** $* *$ *

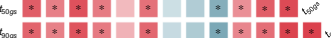

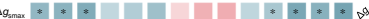

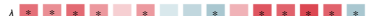

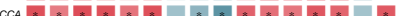

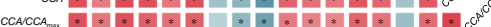

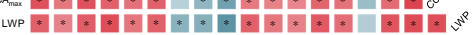

LA

Correlation co 